\title{
Numerical Simulation of Heat and Moisture Coupling of Porous Fabrics
}

\author{
Fengping Ren ${ }^{1} \&$ Shan Cong ${ }^{1}$ \\ ${ }^{1}$ School of Fashion Technology, Shanghai University of Engineering Science, Shanghai, China \\ Correspondence: Renfengping, Address: School of Fashion Technology, Shanghai University of Engineering \\ Science, Shanghai, 201600, China
}

Received: June 26, 2017

Accepted: July 30, 2017

Online Published: August 18, 2017

doi:10.5430/jbar.v6n2p35

URL: https://doi.org/10.5430/jbar.v6n2p35

\begin{abstract}
In this paper, the mechanism of heat and moisture transfer is first described. Then, the development of heat and moisture transfer model and numerical simulation method are summarized. Finally, the existing problems in numerical simulation of heat and moisture transfer in fabrics are analyzed, and the future development of them is prospected.
\end{abstract}

Keywords: Porous fabric, Heat and moisture transfer, Coupled model, Numerical simulation

\section{Introduction}

In recent years, the research on porous media has penetrated into many disciplines and new technologies, including energy, materials, chemical engineering, environmental science, biological technology, bionics, medicine and agricultural engineering (Shi Mingheng, Yu Weiping, Wang Buxuan, 1994). The study of the coupling law of heat and mass transfer in porous media can not only understand the structure and physical and chemical properties of porous media more deeply, but also have extensive practical value.

The porous media in nature are changeable and complex. Generally speaking, porous media can be divided into three categories (Wang Buxuan, 1998): (1) a porous medium, such as a packed grain, two variables metal particle mixture, etc; (2) skeleton porous medium, such as porous rock, timber, well developed soil and so on; (3) fibrous porous media, such as clothes worn on the body.

As textile materials are porous media, it is very feasible to introduce the heat and mass transfer method of porous media into the process of heat transfer and moisture transfer in textile materials. As a traditional industry, clothing and other branches of the integration of innovation is the current research hot spot and direction, but also the inevitable choice for the development of the field of clothing. It is still a concern for scholars and developers to apply the theory of heat and mass transfer in porous media to the field of clothing and study the heat and moisture transfer process of the micro system composed of environment, clothing and human body.

\section{Mechanism Analysis of Heat and Moisture Transfer of Fabrics}

Heat transfer is the process by which heat travels from a high temperature region to a low temperature zone, caused by temperature difference. There are 3 forms of heat transfer in porous fiber fabrics: heat conduction, convection and radiation. Heat conduction is the mode of heat transfer produced by the thermal movement of molecules, atoms, and free electrons in stationary materials. Convection is the mode of heat transfer that results in the relative displacement between the parts of a fluid, resulting in the mixing of cold and hot fluid. Because of the irregular thermal motion of fluid molecules, heat convection must be accompanied by thermal conduction. The object through electromagnetic wave heat transfer is called radiative heat transfer (YANG Shiming, TAO Wenshuan, 2006).

In the actual heat transfer process, the 3 modes of transmission do not exist alone, but can occur simultaneously without depending on each other. The gaps between the porous fiber fabric, yarn between the fibers within or between fibers in yarns is relatively small, in general, the heat conduction in a dominant position, while heat convection and thermal radiation contribute less to (SHI Hongliang, 2001). But at high temperatures, thermal radiation is not to be ignored.

From a macro perspective, the porous fabric as a collection of fiber and air, normally air contains some water. In the course of heat transfer, there will also be the transmission and absorption of water, and simultaneous existence and interaction with heat transfer. The moisture absorption capacity of different fibers is different, and the moisture absorption and heat release of the fabric will lead to changes in fabric temperature. At the same time, the increase of 
temperature will cause water vapor saturation, and the concentration of vapor will become larger, and then the change of evaporation will be caused by (LI Fengzhi, 2004).

\section{Development of Coupling Model of Heat and Moisture}

\subsection{Early Studies}

The researchers have a long history of studying the heat and mass transfer properties of porous media. In 1822 , Fourier deduced Newton's law of cooling and obtained the Fourier equation in which the heat flux was proportional to the temperature gradient, and the coefficient of proportionality is the thermal conductivity of the medium. This equation has become the basic theory of heat transfer, and provides an important theoretical basis for the study of heat transfer in porous media. In 1855, Fick established the relationship between fluid concentration gradient and fluid flux inspired by Ohm's law, called Fick's Law. In 1856, Darcy's experiment on filtration of water by porous media obtained Darcy's Law. The establishment of these three basic laws provides a theoretical tool for the theoretical study of heat and mass transfer in porous media. Researchers in various fields have carried out quantitative analysis of heat and mass transfer phenomena in porous media with different structures and obtained a series of results (Tian Mingwei, 2012).

In many porous materials, heat transfer, moisture transfer and air flow will influence each other. A lot of theoretical models have been put forward. The earliest thing to do in this area is Henry P.S.H..He studied the diffusion of moisture through the pores of the solid and that the solid can absorb or release water vapor. It is assumed that the physical properties of porous media are unchanged under normal climatic conditions. Henry established a one-dimensional transient heat transfer and moisture transfer mathematical model (Lin Ruitai, 1995):

Mass conservation equation

$$
\frac{\partial \rho_{v}}{\partial t}+\frac{\rho}{\varepsilon} \frac{\partial U}{\partial t}=D_{v}
$$

Energy conservation equation

$$
\frac{\partial T}{\partial t}-\frac{h_{l}}{C_{p}} \frac{\partial U}{\partial t}=\alpha \frac{\partial^{2} T}{\partial x^{2}}
$$

and

$$
U=\beta \rho_{v}-\gamma T
$$

In the formula, $\rho_{\mathrm{v}}$ is the density of water vapor; $\varepsilon$ is the porosity of the material; $U$ is the moisture content; $D_{v}$ is the amount of water vapour diffusion; $\alpha$ is the temperature coefficient; $\lambda$ is the thermal conductivity of solid materials; $\rho$ is the density of solid material; $h_{\mathrm{lv}}$ is the latent heat of vaporization; $\mathrm{C}_{\mathrm{p}}$ is the specific heat of solid material at constant pressure; $\mathrm{T}$ is the temperature; $\beta$ and $\lambda$ are the proportionality constant.

Henry further analyzed and solved the above model. Equation (3) is a linear function in which Henry assumed that the moisture content in the medium was the temperature and the density of the water vapor. And the medium and the surrounding air at all times maintained a balance of moisture and heat.

In the study of transient heat and mass transfer in porous media, another famous mathematical model is Luikov equation. The equations describe the unsaturated wet diffusion process without total pressure differential action:

$$
\begin{gathered}
\frac{\partial U}{\partial t}=\frac{\partial}{\partial x}\left(D_{m} \frac{\partial U}{\partial x}\right)+\frac{\partial}{\partial x}\left(D_{t} \frac{\partial T}{\partial x}\right) \\
\frac{\partial T}{\partial t}=\frac{1}{\rho C_{p}} \frac{\partial}{\partial x}\left(\lambda \frac{\partial T}{\partial x}\right)+\frac{r h_{l_{V}}}{C_{p}} \frac{\partial U}{\partial t}
\end{gathered}
$$

In the formula, $\mathrm{D}_{\mathrm{m}}, \mathrm{D}_{\mathrm{t}}$ and $\mathrm{r}$ are the solid diffusion coefficients, thermal diffusivity and phase change factors of solid skeleton respectively.

It is assumed that moisture transfer is similar to heat transfer, and the internal transmission is proportional to the moisture gradient and the temperature gradient. Moisture diffusion acts on heat transfer, while phase transition or endothermic reaction of material is regarded as heat source or heat sink. In the coupling problems, heat absorption or heat release is generally a source or sink, and this heat can not be neglected in hot and humid materials. 
Although the solution of the Luikov equation is quite complicated, it takes into account all forms of bound water and does not limit the wet migration. The Luikov model is still considered a reliable and practical set of equations ( $\mathrm{Li}$ Xikui, Li Rongtao, Zhang Xueshan, 2005). The key to obtain numerical solutions of heat and mass transfer is the need for accurate and reliable thermal and boundary conditions in the equation.

\subsection{Recent Studies}

In 2000, a porous composite soil, Jintu Fan (Jintu Fan, Zhongxuan Luo, Yi Li, 2000) et al first put forward with the adsorption and condensation of textile materials of heat and moisture transfer dynamic model about porous composite materials. In the literature (J. Fan, X. Wen, 2002), a dynamic coupled model of heat and moisture coupling through a fibrous insulating layer was also presented. Evaporation and movement condensation were considered in the model. The model successfully explained the experimental observations of Farnworth, and the numerical results of the model were in good agreement with the experimental results. In the literature (J. Fan, X. Cheng, X. Wen \& W. Sun, 2004), Fan Jintu et al proposed an improved model of coupled heat and moisture transfer in fiber heat insulation layer with phase change and moving condensation.

In the previous model, the movement of moisture in the cotton fabric was considered negligible. But later it was found that this resulted in a large error between the numerical results and the experimental data. In the new model, the authors considered the water movement caused by the pressure gradient, the supersaturated state in the condensation region, and the dynamic moisture absorption and fluid condensation of the fiber material. Compared with the numerical results of the new model, the experimental results were in good agreement with the numerical results. In the literature (Huijun Wu, Jintu Fan, 2008), a heat and moisture transfer model of two ply fabric based on the above dynamic model was proposed. In addition to conduction and radiation, the convection heat transfer between the fabric and the outside environment was also considered.

In recent years, the coupled model of fabric heat and moisture has been developed rapidly, and the consideration of influencing factors has been gradually improved. Zhu (ZHU Q Y, XIE M H, YANG J., 2011) developed a fractal mathematical model of pore size distribution in porous media on the basis of coupled heat and mass. The fractal dimension and voidage parameter were introduced, and the initial conditions and boundary conditions were normalized. The water vapor concentration distribution, the volume fraction of liquid water, the temperature change of porous fiber material and the relative molecular weight distribution of water were simulated.

The continuous emergence of new materials has brought new opportunities for the study of coupled heat and moisture transfer of fabrics. Li Fengzhi et al (Li Fengzhi, Wu Chengyun, Li Yi, 2008) (Zhu Yunfei, 2011) developed a model of heat and moisture coupling for fabrics containing phase change materials, and improved the traditional coupled heat and moisture model. The model considered the influence of the phase transition region on the phase transition, the heat transfer process, the influence of the heating / cooling rate on the characteristic temperature and phase transition heat of the phase change material. It could predict the heat and moisture transfer process in phase change microcapsule fabrics very well. Subsequently, a model of heat and moisture transfer in clothing containing single and multiple types of phase change microcapsules was developed. And it provides a good guidance for the design of smart clothing fabric, but the model dose not take into account the yarn.

At present, many scholars build mathematical models from the point of view of fluid mechanics and thermodynamics to simulate the internal flow field of porous media, and simplify the setting of model and boundary conditions to reduce the modeling time (Zhang Mingbao, 2012).

\section{Calculation of Numerical Simulation}

The numerical calculation of heat and moisture differential equations of porous fiber fabrics is usually based on numerical method. The numerical method can be used to solve the problems of irregular geometry, complicated boundary conditions or nonlinear heat conduction problems. The commonly used numerical methods are the finite element method, the finite volume method, the finite difference method, and the control volume time domain recursive algorithm (XU Dinghua, GE Meibao, ZHANG Haili, 2013). Xu Dinghua et al (XU Dinghua, GE Meibao, 2010) proposed a strong coupled nonlinear equations for heat and moisture coupling. First, the equations were decoupled. Then, the original equation was discretized into a nonlinear system of equations by means of finite difference and numerical integration, and ewton iteration method was used to solve the problem. Then calculated the temperature distribution inside the fabric, the mass flux of water vapor and the pressure of water vapor. The numerical results were in good agreement with the experimental data, which demonstrated the effectiveness and feasibility of the proposed algorithm.

With the continuous improvement of numerical methods and the application of various calculation soft wares, the 
speed and accuracy of numerical simulation have been greatly improved. For example, Matlab can be used to solve the mathematical model by programming language. According to the numerical solution, the distribution of temperature and humidity in the fabric can be plotted over time.

\section{Conclusion and Prospect}

In recent years, the factors considered in the establishment of the coupling model of fabric heat and moisture are more and more close to the actual situation. More applied to real life, provide a scientific basis for the development of new products. The method of solving the model has also been greatly improved, and the speed and accuracy of numerical simulation have also been greatly improved.

At the same time, the established mathematical model also has some limitations. In order to reduce the amount of calculation, the previous studies often simplified model. The assumed conditions, boundary conditions, fabric morphology and properties are mostly idealized. There is a certain deviation between the calculated results and the actual situation. Most of the established models of fabric heat and moisture coupling are based on the micro element model. It is only a preliminary consideration of the influence of the fabric's physical and structural characteristics on heat transfer and moisture transfer (Wang Hongmei, Zheng Zhenrong, Zhang Nannan, 2016). In the future research, it is still necessary to study the heat and moisture transfer mechanism of porous fabrics and establish a perfect numerical model for heat and moisture transfer of fabrics. The establishment of model under unsteady condition is also a research focus. In addition, there are still a lot of problems to be solved for the establishment of the coupled thermal and moisture model of multilayer fabric and complex fabric in the three-dimensional direction, which need the further research.

\section{References}

Huijun Wu, Jintu Fan. (2008). Study of Heat and Moisture Transfer within Multi-layer clothing Assemblies Consisting of Different Types of Battings. International Journal of Thermal Sciences, 47, 641-647. https://doi.org/10.1016/j.ijthermalsci.2007.04.008

J. Fan, X. Cheng, X. Wen \& W. Sun. (2004). An improved model of heat and moisture transfer with phase change and mobile condensates in fibrous insulation and comparison with exper- imental results. Int. J. Heat Mass Transfer, 47, 2343-2352. https://doi.org/10.1016/j.ijheatmasstransfer.2003.10.033

J. Fan, X. Wen. (2002). Modeling heat and moisture transfer. Int. J. Heat Mass Transfer, 45, 4045-4055. https://doi.org/10.1016/S0017-9310(02)00114-X

Jintu Fan, Zhongxuan Luo, Yi Li. (2000). Heat and Moisture Transfer with sorption and condensation In porous clothing assemblies and numerical simulation. Int. J. Heat Mass Transfer, 43, 2989-3000. https://doi.org/10.1016/S0017-9310(99)00235-5

Li Fengzhi, Wu Chengyun, Li Yi. (2008). Study on heat and moisture transfer model of porous fabrics with additional phase change microcapsules. Journal of Dalian University of Technology, 48(2), 162-167.

LI Fengzhi. (2004). Mathematical modeling and numerical simulation to problems of heat and mass transfer in textiles and clothed human body. Dalian: Dalian University of Technology, 9-10.

Li Xikui, Li Rongtao, Zhang Xueshan. (2005). Numerical simulation of coupled thermo hydro mechanical processes in concrete under high temperature. Engineering Mechanics, 22(4), 171-178.

Lin Ruitai. (1995). Heat and mass transfer in porous media. Beijing: Science Press, 53-65.

SHI Hongliang. (2001). The theory discussion of the mechanism of heat transfer in textiles. Journal of NantongUniversity, (4), 44-47.

Shi Mingheng, Yu Weiping, Wang Buxuan. (1994). Current situation and prospect of heat and mass transfer in porous media. Journal of Southeast University. 24, 1-7.

Tian Mingwei. (2012). Analysis of heat transfer properties of multiphase porous fiber materials under unstable state. Shanghai: Donghua University.

Wang Buxuan. (1998). Theory of heat and mass transfer. Science Press.

Wang Hongmei, Zheng Zhenrong, Zhang Nannan. (2016). Research progress of numerical simulation on heat and moisture transfer in porous textiles. Journal of Textile Research, (11), 159-165. 
XU Dinghua, GE Meibao, ZHANG Haili. (2013). Numerical solution of a dynamic model of heat and moisture transfer in porous fabric under low temperature. International Journal of Heat and Mass Transfer, 61, 149-157. https://doi.org/10.1016/j.ijheatmasstransfer.2013.01.045

XU Dinghua, GE Meibao. (2010). A numerical method of a heat and moisture transfer model in textiles. Jiangxi Science, 28(5), 578-580.

YANG Shiming, TAO Wenshuan. (2006). Heat transfer. Beijing, Higher Education Press, 4-12.

Zhang Mingbao. (2012). Thermal simulation method of flow field in porous media. Power Station Auxiliary Equipment, 33(2), 9-12.

ZHU Q Y, XIE M H, YANG J. (2011). A fractal model for the coupled heat and mass transfer in porous fibrous media. International Journal of Heat and Mass Transfer, 54(7), 1400-1409. https://doi.org/10.1016/j.ijheatmasstransfer.2010.12.001

Zhu Yunfei. (2011). Model of heat and moisture transfer in phase change clothing and model study on the influence of thermal response on human body. Nanjing: Nanjing University of Aeronautics \& Astronautics. 